\title{
Corela
}

Cognition, représentation, langage

HS-19 | 2016

Le point de vue pris au mot

\section{Introduction : la Sémantique des Points de Vue}

\section{Zsófia Várkonyi}

\section{OpenEdition}

\section{Journals}

Édition électronique

URL : http://journals.openedition.org/corela/4268

DOI : $10.4000 /$ corela.4268

ISSN : $1638-573 X$

Éditeur

Cercle linguistique du Centre et de l'Ouest - CerLICO

\section{Référence électronique}

Zsófia Várkonyi, «Introduction : la Sémantique des Points de Vue », Corela [En ligne], HS-19 | 2016, mis en ligne le 08 juin 2016, consulté le 01 mai 2019. URL : http://journals.openedition.org/corela/4268 ; DOI : $10.4000 /$ corela.4268

Ce document a été généré automatiquement le 1 mai 2019.

\section{(c) (i) (2)(2)}

Corela - cognition, représentation, langage est mis à disposition selon les termes de la licence Creative Commons Attribution - Pas d'Utilisation Commerciale - Partage dans les Mêmes Conditions 4.0 International. 


\title{
Introduction : la Sémantique des Points de Vue
}

\author{
Zsófia Várkonyi
}

1 Pierre-Yves Raccah est un chercheur qui développe ses idées jusqu'à leurs termes. D'une rigueur implacable sur le plan de la méthode, il a acquis la réputation d'être un auteur radical. En effet, sa démarche intellectuelle sans compromis l'a poussé à rompre avec un certain nombre de dogmes scientifiques et à concevoir son propre système. Ce système se base sur des conceptions originales, notamment concernant la nature de la construction du sens et les contraintes linguistiques qui régissent cette dernière.

2 Ce perfectionnisme caractérise également son approche des autres auteurs. Son travail s'inscrit dans la lignée d'une tradition linguistique qui part de Saussure et passe par Ducrot, en systématisant, formalisant, et poussant souvent plus loin les idées qu'il a incorporées. Le choix de ses dernières n'a pas à voir avec ce qui est en vogue, ce qui est répandu, ce qui est généralement considéré comme incontournable, mais seulement avec ce qui trouve sa place dans son système. Ce deuxième fait explique que malgré la radicalité des concepts qu'il utilise, Raccah étonne par son habitude de revenir à des idées ou modèles déjà délaissés par d'autres. Pas d'innovation à tout prix - bien qu'il ait joué un rôle actif dans l'élaboration du concept de topos lexical et ouvert de nouvelles pistes dans la description sémantique formelle des unités lexicales - mais, avant tout, une prise au sérieux des idées pertinentes.

3 Ainsi, si Pierre-Yves Raccah ne prétend pas avoir introduit la notion de point de vue en linguistique, c'est bien lui, et lui seul, qui a mis en évidence le fait qu'un groupe bien délimité de phénomènes sémantiques, phénomènes qui sont décrits aux moyens de modèles hétéroclites, peut être décrit à l'aide du seul concept technique de point de vue. La Sémantique des Points de Vue, qui réunit et systématise ces modèles, s'écrit désormais avec des majuscules et porte la signature de Raccah. 


\section{D'une épistémologie à une terminologie, en passant par une méthodologie}

Il serait difficile de tenter de récapituler les tenants et aboutissants de la Sémantique des Points de Vue sans faire le point sur quelques-uns des termes de base de cette dernière. La linguistique est un domaine où chaque auteur doit trouver un compromis entre l'utilisation des termes conventionnels, censés garantir l'intercompréhension entre chercheurs, et l'introduction de nouveaux termes, ou encore des nouvelles définitions de certains anciens termes, que la spécificité de chaque théorie ou modèle rend nécessaire. Même les termes les plus fondamentaux de la linguistique ont parfois de nombreuses acceptions en fonction des auteurs, suivant les phénomènes dont ils doivent rendre compte et les concepts qu'ils doivent manipuler. Dans le cas que l'on pourrait qualifier d'idéal, le choix des termes est fortement conditionné par une réflexion épistémologique, préalable à tout travail scientifique. Les bases épistémologiques d'une théorie touchent généralement aux points suivants : la scientificité, l'empiricité de la théorie et la nature des faits dont elle rend compte. Ces aspects sont imbriqués et difficiles à séparer : une prise de position relative à l'un des aspects entraîne des choix dans un autre, et l'ensemble de ces choix épistémologiques va déterminer une partie importante de la méthodologie.

5 Les problèmes d'ordre épistémologique de la linguistique, d'après Raccah, sont très peu spécifiques à cette discipline particulière, et tout aussi peu spécifiques aux sciences humaines en général. Selon lui (2005: 52-64), malgré les objets apparemment très différents, les sciences humaines sont logées à la même enseigne que les sciences dites dures, à la fois du point de vue des critères de scientificité et du point de vue de leurs limites empiriques. La linguistique, y compris la sémantique, n'a pas de statut particulier. D'une part, malgré la nature subjective des phénomènes qu'elle étudie, elle ne peut pas s'affranchir d'une objectivité dans ses raisonnements scientifiques: une démarche déductive est préférée à une démarche inductive, conformément aux préconisations d'Auroux (1988: 52-54). D'autre part, les sciences dures sont tout aussi confrontées à l'impossibilité de l'observation directe et sans préjugés que les sciences humaines ${ }^{1}$.

6 La position de Raccah (1987, 2005a, 2005c, 2006 etc.) sur les points concernant la méthodologie est proche, à bien des égards, de celle d'Auroux (1998) et de Labov (2001 [1975]). Si l'on accepte avec Labov (2001[1975] : 2) qu'en linguistique, les phénomènes variables doivent être d'abord distingués des invariants, et que c'est à partir des invariants (autrement dit, d'un niveau d'abstraction rendant possible la généralisation) que l'on parvient à comprendre les phénomènes variables observés, il est indispensable de réserver des termes respectifs à ces deux aspects. Ainsi, d'une part, les phénomènes observables, qui sont uniques, variables et fortement dépendants de la situation, sont les discours, constitués d'énoncés qui, eux, peuvent être décomposés en mots-d'énoncés. D'autre part, le niveau d'abstraction correspondant à ces composants est celui de la langue, des phrases et des mots-de-phrases. De la même manière, une sémantique qui se veut générale doit faire la distinction entre le sens des énoncés (variable et dépendant de la situation) et ce qui, au niveau purement linguistique, est responsable de ce dernier : la signification.

7 Selon Raccah, le sémanticien fait face à un défi d'empiricité. Il découle des distinctions esquissées ci-dessus que l'objet théorique de la sémantique est la signification des phrases et des mots-de-phrases. Comme le rappelle Raccah régulièrement dans ses textes, le problème 
que rencontre le sémanticien qui se dispose à faire un travail scientifique empirique est le fait que son objet n'est pas directement observable. Même en faisant correspondre à ces entités abstraites que sont les phrases et leurs significations leurs manifestations dans la réalité empirique : les énoncés et leur sens, il reste le problème que le sens n'est toujours pas accessible à l'observateur. Ce qui est accessible, ce sont les énoncés - en tous cas, leur aspect matériel - et certains de leurs effets, dont certains sont dus à leur sens. Le travail du sémanticien commence donc par l'hypothèse de travail que voici :

Pour le sémanticien, la phrase, qui, rappelons-le, est une catégorie de forme d'énoncé, se laissera observer indirectement à partir de l'observation plus directe des énoncés ; quant au sens, il ne pourra être observé que par ses traces comportementales : toute sémantique empirique s'appuie sur un postulat d'attribution causale permettant d'associer un comportement observé à l'effet d'un énoncé observé. (Raccah $2006: 125$ )

\section{Tous les noms des points de vue}

La thèse centrale de la Sémantique des Points de Vue (dorénavant SPV) est que les mots d'une langue comportent, dans leur signification, des contraintes sur la manière dont l'interlocuteur doit voir ces mots, et / ou d'autres mots. L'idée, formulée ici de manière concise mais un peu obscure devient facilement plus claire à travers quelques exemples, et, je l'espère, parfaitement limpide après un certain nombre de développements théoriques.

Pour reprendre un des exemples favoris de Raccah ${ }^{2}$ : un énoncé de la phrase

John is a republican but he is honest

ne peut être compris qu'à condition qu'on tienne compte de deux types de contraintes sur les points de vue. La première contrainte consiste à obliger l'interlocuteur d'adopter des points de vue opposés sur "republican» et "honest». Cette contrainte est due à la signification de «but » qui présuppose cette opposition ${ }^{3}$, quels que soient les segments de phrase qui le précèdent et qui le suivent. La deuxième contrainte est directement fournie par le mot «honest». Il fait partie d'un groupe de mots qui contiennent en leur signification une évaluation positive, indépendante de toute situation. Ainsi, l'interlocuteur est obligé de voir, du moins le temps de comprendre l'énoncé, « republican »- un mot qui est par ailleurs exempt de jugement et définissable par des critères objectifs - de manière négative.

11 Après avoir présenté, réunis dans une seule phrase, un cas de contrainte sur la forme des points de vue («but ») et un cas de contrainte sur la nature des points de vue (« honest $)$, considérons un autre exemple. Le mot «maison", l'affirme Raccah", cristallise dans sa signification un point de vue lié à l'agrément. Cette affirmation plutôt contre-intuitive se voit justifiée par le test suivant : si ce point de vue n'était pas présent dans la signification du mot, rien n'expliquerait l'étrangeté de la phrase

?? C'est une maison mais elle est agréable

et la « normalité » de la phrase

C'est une maison mais elle n'est pas agréable.

Ce point de vue cristallisé (c'est-à-dire, inscrit de manière stable) dans la signification de « maison» n'est pas réductible à une simple évaluation positive ou négative, comme dans le cas de «honest ». 
14 Ces observations ne concernent pas seulement quelques cas isolés mais des phénomènes bien plus vastes, d'où la nécessité de passer à un niveau plus général, niveau à partir duquel on peut élaborer des descriptions sémantiques, applicables à d'autres cas. En effet, la SPV fait correspondre ces points de vue, présentés jusqu'ici de manière informelle, à des concepts techniques qui sont définis dans le cadre de la théorie :

- Les points de vue, qu'ils soient élémentaires ou complexes, sont décrits au moyen du concept de champ topique lexical 5 . La SPV rend compte des points de vue élémentaires à travers le concept de la phoricité: une partie du lexique de chaque langue se classe dans la catégorie des mots qui sont soit euphoriques, soit dysphoriques. Il s'agit de mots qui charrient, en toute situation, une évaluation positive, pour les euphoriques, et négative, pour les dysphoriques.

16 - La description des articulateurs (connecteurs et opérateurs) s'effectue en termes de contraintes sur la forme des topoi.

17 - La SPV inclut également le modèle polyphonique, parallèlement avec le modèle topique. C'est une autre notion de point de vue qui est mobilisée ici, celle qui se rapporte à l'énonciation. Certaines descriptions nécessitent l'attribution des points de vue - des sortes de prises de position - à des énonciateurs abstraits.

18 Ces termes techniques et les concepts qu'ils nomment proviennent, pour la plupart, des travaux plus anciens effectués dans le cadre de l'Argumentation dans la Langue (AdL) ${ }^{6}$. Je propose un zoom sur ces concepts qui sont cruciaux pour la compréhension des travaux de Raccah, ainsi que de ceux des autres auteurs qui utilisent les modèles de la SPV.

\section{L'héritage de l'AdL}

19 Raccah se définit, quasiment depuis ses débuts en sémantique et jusqu'à nos jours, comme un disciple d'Oswald Ducrot. Si, ce que l'on peut appeler «l'école ducrotienne » se représente aujourd'hui sous forme de plusieurs sous-écoles, c'est parce que Ducrot est un chercheur qui n'a jamais hésité à se remettre en question, revenir sur ses anciennes idées et changer de modèle théorique quand cela lui a semblé nécessaire. Il existe donc plusieurs modèles issus de ses travaux, modèles dont les plus anciens ont été abandonnés par leur concepteur, mais qu'un certain nombre de chercheurs estiment encore dignes d'intérêt. C'est ainsi que Raccah peut être un ducrotien qui ne marche pas tout à fait dans les pas de Ducrot...

20 Le dénominateur commun de toutes ces sous-écoles reste la thèse de l'argumentation dans la langue, qui peut se résumer de la manière suivante :

[...] même si tous les énoncés ne sont pas nécessairement des argumentations, il n'en reste pas moins que toute phrase peut être utilisée dans un énoncé qui est une argumentation. Une conséquence théorique essentielle de ce fait est qu'une description sémantique des phrases (et donc de leurs constituants) doit nécessairement rendre compte de leurs utilisations dans des énoncés qui constituent des argumentations : une théorie adéquate de la signification doit, au minimum, comporter un volet argumentationnel.» (Raccah $2002: 241$ )

21 La différence consiste en la manière dont elles rendent compte de cette potentialité argumentative. Le cadre de cette Introduction ne permet pas la présentation des modèles des échelles argumentatives ${ }^{7}$ et des blocs sémantiques ${ }^{8}$, seul le modèle topique ${ }^{9}$ sera présenté. Ce dernier ne comporte pas les inconvénients que présentent les échelles - et ne comporte pas non plus les prétendus inconvénients pour lesquels il a été délaissé. 


\subsection{Les topoi} telles, des principes d'inférence, propres à une communauté linguistique servent de garants. Ils assurent le passage entre les arguments et les conclusions que ceux-ci étayent. Les argumentations possibles sont délimitées par le choix des mots, qui, eux-mêmes, évoquent certains de ces principes. Ces thèses sont habituellement illustrées par l'exemple suivant, devenu emblématique :

Il fait beau mais j'ai du travail.

Où la situation d'énonciation est une proposition de promenade à laquelle cet énoncé est la réponse. Le premier segment de l'énoncé (Il fait beau) est, dans la situation donnée, un argument en faveur de la promenade, le deuxième segment (j'ai du travail) est un argument contre la promenade ${ }^{10}$. Les garants qui permettent de comprendre en faveur de quelles conclusions ces segments sont destinés correspondent aux principes :

g1) « le beau temps est favorable à la promenade »

g2) « le travail est défavorable à la promenade ».

G1 et g2 sont des formulations possibles des principes qui sont généralement acceptés par une communauté linguistique. Comme, en général, ces principes ne sont pas explicités dans le discours, la manière précise dont ils sont formulés n'a pas beaucoup d'importance. Tous ces principes, appelés topoi ${ }^{11}$ en référence à la notion aristotélicienne, ont trois caractéristiques en commun: ils sont présentés comme généraux (c'est-à-dire comme applicables à d'autres situations que celle de l'énonciation), présentés comme partagés (c'est-à-dire comme acceptés par une communauté dont l'interlocuteur fait également partie), et sont graduels, même si les énoncés s'appuyant sur les topoi ne sont pas formulés de façon graduelle.

25 En ce qui concerne les deux premières caractéristiques, les topoi ne sont pas nécessairement généraux et partagés, mais ils sont toujours présentés comme tels, sans quoi ils ne pourraient pas servir de garant. Le caractère graduel des topoi, en revanche, découle de leur nature. Bruxelles, Ducrot, Raccah (1995: 100) présentent l'exemple suivant pour illustrer cette thèse :

Les énoncés de la phrase "Il a certainement gelé cette nuit, car les plantes sont mortes » s'appuient sur le principe qui établit un lien entre le gel et la mort des plantes. Les énoncés de la phrase « Il a dû faire très froid cette nuit, car les plantes sont en mauvais état » évoquent une autre règle, reliant le froid avec le mauvais état des plantes. Ces deux règles portent sur des degrés distincts des mêmes propriétés (degré de froid, degré de mauvais état). Les topoi étant des généralisations de ce type de garants d'argumentation individuels, ils sont eux-mêmes graduels. Le topos généralisant ces deux garants (et d'autres, figurant sur la même échelle) serait

//Plus il fait froid, plus les plantes sont en mauvais état.//

Le topos est défini, et sa nature ainsi explicitée par Raccah :

Un topos est une catégorie de garants d'argumentation, indiquant la prise en compte d'une corrélation entre deux entités. [...] La prise en compte de la corrélation entre les deux entités est discursive: rien n'empêche un locuteur d'admettre un topos dans un discours et d'admettre le topos contraire dans un autre. (Raccah 2005a: 186) 
La version topique de l'AdL défend l'hypothèse selon laquelle tous les topoi peuvent être réduits à la même forme. Les topoi sont formalisés de la façon suivante :

Si l'énoncé $\mathrm{E}$ indique qu'une entité $\mathrm{X}$ possède une propriété quelconque $\mathrm{P} 1$ et si ce fait permet (au locuteur) d'évaluer $X$ dans une échelle $P$, appelée champ topique de l'énoncé $\mathrm{E}$, alors les topoi applicables à $\mathrm{E}$ ont l'une des formes suivantes :

// plus $\mathrm{x}$ est $\mathrm{P}$, plus y est $\mathrm{Q} / /$

// plus $x$ est $P$, moins y est $Q / /$

$/ /$ moins $x$ est $P$, plus y est $Q / /$

// moins $x$ est $P$, moins y est $Q / /$. (Raccah $1990: 182)$

On utilise également la formulation courte

$/ /+\mathrm{P},+\mathrm{Q} / / / /-\mathrm{P},-\mathrm{Q} / /$

$/ /+\mathrm{P},-\mathrm{Q} / / / /-\mathrm{P},+\mathrm{Q} / /$

où $\mathrm{P}$ et $\mathrm{Q}$ sont des champs topiques, + et - sont les signes des champs topiques. +/-P est appelé l'antécédent $d u$ topos, $+/-\mathrm{Q}$ le conséquent $d u$ topos. Le locuteur ou l'interlocuteur qui admet //+P, $+\mathrm{Q} / /$ doit nécessairement admettre //-P, $-\mathrm{Q} / /$. En d'autres termes, ces deux formes illustrent la même idéologie ou manière de voir le monde. Symétriquement, celui qui admet //+P, $-\mathrm{Q} / /$ doit aussi admettre //-P, $+\mathrm{Q} / /$, même si les deux formes distinctes de la même idéologie ont des rôles différents dans leurs actualisations linguistiques.

1 On a pu remarquer que ces topoi qui régissent le discours argumentatif (et qui, pour cette raison, seront appelés topoi discursifs ou topoi dynamiques, à la différence des topoi lexicaux, présentés plus loin), ne sont pas des entités linguistiques. De ce fait, il n'appartient pas à la sémantique de tenter d'inventorier les topoi utilisés par une communauté linguistique. Comme l'affirment Bruxelles, Ducrot et Raccah (1995: 100), ce répertoire de topoi incombe davantage à l'anthropologie culturelle. En revanche, la description sémantique des unités linguistiques comporte des indications stables sur la manière dont ces unités contraignent l'utilisation des topoi.

\subsection{Les travaux sur les articulateurs}

Les connecteurs (articulateurs qui relient deux segments de phrase) et les opérateurs (articulateurs qui agissent sur un segment de phrase) ont ceci de particulier que d'un point de vue vériconditionnel, il est généralement impossible de rendre compte de certains effets de sens systématiques qu'ils entraînent. En supprimant ou en modifiant les articulateurs dans un discours, le sens de ce dernier serait également, nécessairement modifié. En considérant que les instructions stables que ces unités linguistiques donnent sur la manière dont on doit interpréter les énoncés constituent leur signification, l'AdL a ouvert la voie vers une description sémantique qui rend compte de ces instructions. Dans la période qui précédait la TT, cela s'effectuait en termes d'arguments et de conclusions, ensuite, en termes de contraintes sur la forme des topoi évoqués. Cette méthode permet de proposer des descriptions qui ne dépendent ni de la situation d'énonciation ${ }^{12}$ réelle des énoncés, ni des topoi effectivement évoqués. La description de " mais $»^{13}$, par exemple, rend compte du fait que dans toute situation, et quel que soit le topos sur lequel l'argumentation de l'énoncé comportant «mais » se base, la forme du conséquent du topos mobilisé par le segment A est l'inverse de la forme du conséquent du topos mobilisé par le segment $B$. Le conséquent du topos mobilisé par l'énoncé $A$ mais $B$ est identique au conséquent du topos mobilisé par B. 

rendu possible la description sémantique des mots «ordinaires». Il en a résulté que la description des articulateurs, si elle n'a pas été tout à fait reléguée au second plan, n'a pas été pour autant systématiquement mise à jour à la lumière des résultats théoriques plus récents. Des descriptions formelles en termes de contraintes sur les topoi ont été néanmoins réalisées pour plusieurs connecteurs et opérateurs, à titre d'exemple, « mais » (Raccah 1990 : 29-31), « trop » (Raccah 2005a), « assez» (Raccah, Várkonyi 2012).

\subsection{Les topoi lexicaux}

La description des articulateurs met en évidence des contraintes sur la forme des topoi mobilisés dans les énoncés. Or, les contraintes linguistiques peuvent concerner la nature même de ces derniers, lorsqu'ils se cristallisent dans le lexique, selon la thèse de la théorie des topoi lexicaux (Bruxelles, Ducrot, Raccah 1995), qui a directement conduit, plus tard, à la conception de la SPV.

[...] les mots choisis contraignent les topoi possibles (ou encore, du point de vue opposé, les topoi que l'on veut utiliser contraignent le choix des mots) : n'importe quel topos ne peut pas être associé à n'importe quelle phrase. On pourrait parler ici de sous-détermination, dans un sens positif (c'est-à-dire, dans un sens où sousdéterminer implique un certain degré de détermination). (Raccah $1998: 12$ )

Le concept du champ topique lexical (ou topos intrinsèque à un mot, selon une terminologie plus ancienne) est issu de l'hypothèse selon laquelle certains mots cristallisent des topoi dans leur signification. Ainsi, lorsqu'un énoncé contient un mot qui cristallise un topos, les potentialités argumentatives de l'énoncé en sont en partie déterminées. L'exemple le plus fréquemment cité pour illustrer cette thèse est celui du mot «riche ». Dans l'article de 1994, cité plus haut, Bruxelles et alii. démontrent que ce mot active systématiquement un topos qui relie la possession à la capacité d'agir (//Plus on possède, plus on peut agir// ). Le mot « riche» ne faisant pas partie des euphoriques ou dysphoriques, une évaluation positive ou négative peut l'accompagner.

Selon la modélisation de ce phénomène de cristallisation, les champs topiques (CT) qu'on peut associer aux mots sont décomposables en chaînes de champs topiques, ce qui permet de rendre compte du cheminement des connaissances, des idéologies ou des croyances propres à une communauté linguistique qui se cristallisent dans le lexique de cette communauté. Le résultat de cette décomposition est le couple constitué d'un champ conceptuel (CC) et d'un autre champ topique, qui est également décomposable. La fin d'une série de décomposition est un couple qui se compose d'un $\mathrm{CC}$, et d'un champ topique élémentaire (CTe). Le CTe est constitué d'un champ conceptuel et d'un point de vue (positif ou négatif) sur ce champ conceptuel:

Un champ topique est, en gros, une «façon de voir » une entité, une propriété ou une relation. Cette façon de voir est, elle-même, déterminée par la façon dont on voit une autre entité, une autre propriété ou une autre relation: c'est à dire par un autre champ topique. On peut ainsi représenter un champ topique par une chaine de champs topiques emboités les uns dans les autres, de telle sorte que chaque champ topique est caractérisé d'une part, par un champ conceptuel (l'entité, la propriété ou la relation), et d'autre part, par le champ topique qu'il contient, lequel est lui-même caractérisé par un champ conceptuel et par le champ topique qu'il contient, et ainsi de suite jusqu'à un champ topique élémentaire. Ce dernier étant un principe de valuation, introduit une gradation dans le champ topique qui le contient. (Raccah 1998 : 12-13)

Corela, HS-19 | 2016 
Ainsi, le CT associé au mot français « riche » peut être décrit comme la possession (CC) vue du point de vue du pouvoir (CT), ce dernier étant lui-même constitué d'un jugement, positif ou négatif, sur la capacité d'agir. La description formelle en est une des suivantes :

riche : <POSSESSION, <CAPACITÉ D'AGIR, + >>.

riche : <POSSESSION, <CAPACITÉ D'AGIR, - >>.

Ce dispositif de description réunit deux avantages majeurs. D'une part, il permet un traitement formel des mots, sans avoir recours à un réductionnisme logique. D'autre part, il rend compte de l'inscription des certains aspects socioculturels dans le lexique, tout en restant à un niveau sémantique donc strictement linguistique.

\subsection{Le modèle polyphonique}

Bien qu'il ne s'agisse pas d'un héritage dans le sens propre du mot, il convient de mentionner le modèle polyphonique dans ce chapitre. Le concept bakhtinien de polyphonie a inspiré un bon nombre de travaux au sein de diverses disciplines: plus directement en études littéraires et en analyse de discours, indirectement en linguistique.

La notion de polyphonie apparaît chez Ducrot dans ses travaux antérieurs à l'AdL, mais il le reprend également dans (Anscombre, Ducrot $1983: 174-179$ ). Les auteurs consacrent un chapitre à la combinaison des deux systèmes (polyphonique et argumentatif). Raccah retient des travaux ducrotiens de cette époque la distinction entre sujet parlant, locuteur et énonciateur. Le sujet parlant est l'être du monde (qui, en tant que tel, n'intéresse pas le linguiste), le locuteur est le responsable de toute l'énonciation, tandis que les énonciateurs "émettent des voix", non pas toujours assumées, à l'intérieur de l'énonciation. La distinction du sujet parlant et du locuteur est pertinente dans la mesure où les deux ne coïncident pas dans tous les cas (par exemple, dans les discours rapportés en style direct).

41 Depuis le début des années 2000 (au cours de son séminaire à L'ILPGA) et depuis 2005 dans ses articles également, Raccah plaide pour l'intérêt d'une conception polyphonique pour la langue ${ }^{14}$. Alors que le modèle des champs topiques lexicaux permet d'étudier les contraintes sur les points de vue, « la polyphonie dans la langue, quant à elle, décrit les contraintes sur les attributions de points de vue et sur l'attitude du locuteur vis-à-vis des énonciateurs qu'il évoque.» (Raccah 2005c: 218). Cette idée rejoint "la remarque de Benveniste selon laquelle les énoncés contiennent des traces de leurs conditions d'énonciation" (Raccah 2005c: 209). L'article cité comporte un certain nombre d'illustrations de ce phénomène.

\section{Travaux sur les applications en sciences cognitives}

Si le but principal de cette Introduction est de donner un aperçu de la Sémantique des Points de Vue en tant que prolongement de la Théorie des Topoi, il serait d'une négligence impardonnable de ne pas mentionner les travaux de Pierre-Yves Raccah sur les applications du modèle topique en sciences cognitives. Deux ouvrages collectifs sous sa direction $(1996,2005)$, ainsi qu'un bon nombre de ses articles ont été consacrés à des travaux théoriques destinés à ouvrir des pistes vers ce type d'applications.

43 Raccah est connu pour son approche critique vis-à-vis de ce qu'il appelle le « réductionnisme cognitif», c'est-à-dire l'assimilation de l'étude du langage (faculté 
cognitive) à celle des langues (systèmes abstraits). Cette position critique face à la linguistique cognitive américaine ne cache pas pour autant un refus catégorique de s'intéresser à la cognition, dans la mesure où selon son hypothèse, les observables linguistiques peuvent renseigner sur la façon dont l'intelligence humaine gère les connaissances. Cette hypothèse de l'abstraction cognitive a été formulée ainsi :

La langue est un produit de l'esprit humain et on peut penser que ses structures sont des manifestations de structures plus abstraites de cet esprit, structures dont d'autres manifestations, distinctes des premières mais néanmoins analogues, se retrouvent dans l'usage de la langue et, plus généralement, dans la manière dont l'esprit gère les connaissances. Ainsi, les structures de l'expression linguistique (considérée comme un produit, et non comme une production) sont la trace, dans le domaine de la langue, de structures plus abstraites (appelons-les cognitives) [...]. (Raccah 1999 : 20)

Le noyau de l'hypothèse est une possibilité d'exploitation du modèle topique dans les sciences cognitives. Si la langue se construit sur les topoi, on peut supposer - et il existe de nombreux éléments qui corroborent cette supposition - que c'est un reflet du fonctionnement de l'esprit. Cela a ouvert des pistes vers des travaux d'application, notamment dans le domaine de l'intelligence artificielle.

\section{Pour conclure (et pour mieux introduire)...}

Un recueil d'hommage à Pierre-Yves Raccah n'est bien sûr qu'un prétexte pour faire un petit point - provisoire - sur ces plus de trente ans de sémantique, et pour réunir le présent bouquet d'articles, écrits par ses collègues et ses disciples qui ont souhaité lui rendre hommage. Qu'il s'agisse de chercheurs qui utilisent son cadre théorique, ou qui dialoguent avec celui-ci, ou qui trouvent simplement un parallèle entre leurs champs d'étude respectifs, les auteurs de ce recueil expriment, par leurs contributions, leur estime pour un scientifique d'exception.

\section{BIBLIOGRAPHIE}

Anscombre Jean-Claude, Ducrot Oswald, L'argumentation dans la langue, Liège, Pierre Mardaga, 1983.

Auroux Sylvain, La raison, le langage et les normes. Paris, Presses Universitaires de France, 1998.

Bruxelles Sylvie, Ducrot Oswald, Raccah Pierre-Yves, « Argumentation and the lexical topical fields ", Journal of Pragmatics, ${ }^{\circ} 24: 1 / 2,1995$, pp. 99-104.

Carel Marion, « Trop : argumentation interne, argumentation externe et positivité », in Anscombre Jean-Claude (ed.), Théorie des topoi, 1995, pp. 177-206.

Carel Marion, « Polyphonie et argumentation », in Birkelund Merete, Mosegaard Hansen, Norén Coco (eds), L'énonciation dans tous ses états, Bern, Peter Lang, 2008, pp. 29-45. 
Carel Marion, Ducrot Oswald, « Mise au point sur la polyphonie », Langue française, $\mathrm{n}^{\circ} 164,2009$, pp. 33-44.

Ducrot Oswald, Les échelles argumentatives, Paris, Minuit, 1980.

Ducrot Oswald, « Topoi et formes topiques », Bulletin d'études de linguistique française, $n^{\circ} 22,1988$, pp. 1-14.

Ducrot Oswald, «Quelques raisons de distinguer "locuteurs" et "énonciateurs" » [Article de travail en ligne consulté le 03/03/2013], 2001. Disponible sur internet : http://www.hum.au.dk/ romansk/polyfoni/Polyphonie_III/Oswald_Ducrot.htm

Ducrot Oswald, « Note sur Benveniste et la polyphonie », Béguelin Marie-José, Corminboeuf Gilles (eds), Du système linguistique aux actions langagières. Mélanges en l'honneur d'Alain Berrendonner, Bruxelles, De Boeck / Duculot, Collection Champs linguistiques, 2011, pp. 389-399.

Labov William, « Qu'est-ce qu'un fait linguistique? », Marges linguistiques $\mathrm{n}^{\circ}$ 1, 2001, pp. 25-68, trad. « What is a linguistic fact? », Lisse, The Peter de Ridders Press, 1975.

Popper Karl R., La connaissance objective, Paris, Champs / Flammarion, 1998, trad. Jean-Jacques Rosat, Objective Knowledge: An Evolutionary Approach, Oxford, Clarendon Press, 1972.

Raccah Pierre-Yves, « Sémantique épistémique et loi de prédominance de l'argumentation », Cahiers de Linguistique Française, n 7, 1987, pp. 93-113.

Raccah Pierre-Yves, « Modelling argumentation and modelling with argumentation », Bruxelles, Argumentation, $n^{\circ} 4,1990$, pp. 447-483. [Version en ligne consultée le 16/01/2013]. Disponible sur Internet : http://linguistes.fr/public/Raccah/Publications/Modeling.pdf.

Raccah Pierre-Yves (ed.), Topoi et gestion des connaissances, Paris, Masson, 1996.

Raccah Pierre-Yves, "L'Argumentation sans la preuve : prendre son biais dans la langue », Interaction et cognition, Vol. II, $\mathrm{n}^{\text {os }} 1-2,1998$, pp. 237-264. [Version en ligne consultée le 16/01/2013]. Disponible sur Internet : http://linguistes.fr/public/Raccah/Publications/ biais-1998.pdf.

Raccah Pierre-Yves, « Science, cognition et sémantique des langues », CNRS Working Paper, 1999. Raccah Pierre-Yves, «Lexique et idéologie : les points de vue qui s'expriment avant qu'on ait parlé ", in Carel Marion (ed.), Les Facettes du dire, Hommages à Oswald Ducrot, Paris, Kimé, 2001, pp. 241-268.

Raccah Pierre-Yves, « Une description de l'excessivité en sémantique des points de vue ", Travaux de linguistique du CERLICO, $\mathrm{n}^{\circ}$ 18, 2005a, pp. 171-190.

Raccah Pierre-Yves (ed.), Signes, langues et cognition, Paris, L'Harmattan, $2005 \mathrm{~b}$.

Raccah Pierre-Yves, « Une sémantique du point de vue : de l'intersubjectivité à l'adhésion », Discours Social, $n^{\circ}$ spécial «L'Énonciation identitaire : entre l'individuel et le collectif», 2005c, pp. 205-242.

Raccah Pierre-Yves, « Polyphonie et argumentation : des discours à la langue (et retour...) », in Simonffy Zsuzsa (ed.), L'un et le multiple, Budapest, Tinta Könyvkiadó, 2006, pp. 120-152.

Raccah Pierre-Yves, « Description sémantique du lexique. Un moyen pour seconder efficacement l'intuition », à paraître in Debrenne Michèle (ed.), Limoges, Lambert-Lucas.

Raccah Pierre-Yves, Várkonyi Zsófia, « "Assez", c’est presque "trop" », in Neveu Franck, Muni Toke Valelia, Blumenthal Peter, Klingler Thomas, Ligas Pierluigi, Prévost Sophie, Teston-Bonnard 
Sandra (eds), $3^{e}$ Congrès Mondial de Linguistique Française, Lyon, 4-7 juillet 2012, ILF / EDP Sciences, (Publié sur CD ROM), 2012.

\section{NOTES}

1. Voir Popper (1998[1972] : 231-232) sur ces questions.

2. Raccah (2005a : 180).

3. La nature de cette opposition sera explicitée plus loin.

4. Raccah (2013), à paraître.

5. La définition de ce dernier suppose celle du topos, qui se définit dans le cadre de l'Argumentation dans la Langue. La section 3 de cette Introduction comportera un rappel des notions fondamentales de celle-ci, ainsi qu'une présentation plus détaillée du champ topique lexical, principal outil de description de la SPV.

6. Ducrot (1980) ; Anscombre, Ducrot (1983)

7. Ducrot (1980).

8. Carel (1995).

9. Appelé aussi la Théorie des Topoi (TT). Ducrot (1988).

10. En raison du « mais » qui les relie, l'interlocuteur comprendra l'énoncé entier comme un refus à la proposition de promenade (Anscombre, Ducrot, 1983).

11. Le choix de ce terme technique, désignant un outil conceptuel qui n'a pas beaucoup à voir avec les topoi aristotéliciens, a eu comme conséquence regrettable l'amalgame que l'on a tendance à faire entre les deux concepts.

12. Néanmoins, une situation type est parfois spécifiée, ce qui permet de délimiter un certain emploi de l'articulateur, emploi qui requiert une description spécifique.

13. Cette description concerne le « mais» dit carré.

14. Parallèlement avec Raccah, Ducrot a également retrouvé de l'intérêt dans l'analyse polyphonique et l'a intégrée dans la TBS, dont témoigne (Ducrot 2001). En 2011, il critique son ancienne position qui incluait l'énonciateur dans la description du sens et soutient la Polyphonie dans la Langue (PDL) de (Carel 2008) et (Carel, Ducrot 2009). Anscombre, de son côté, reprend cette notion et l'utilise dans ses travaux depuis 2005. Le cadre de cette Introduction ne permet pas de faire le tour de ces concepts à la fois proches et divergents, je me borne à présenter la manière dont la SPV exploite la conception de départ.

\section{AUTEUR}

\section{ZSÓFIA VÁRKONYI}

Université d'Orléans, LLL 